\title{
Constructing de Sitter vacua in no-scale string models without uplifting
}

\author{
Laura Covi ${ }^{a}$, Marta Gomez-Reino ${ }^{b}$, Christian Gross $^{c}$, \\ Gonzalo A. Palma ${ }^{d}$, Claudio A. Scrucca ${ }^{e}$ \\ ${ }^{a}$ Theory Group, Deutsches Elektronen-Synchrotron DESY, \\ D-22603 Hamburg, Germany \\ ${ }^{b}$ Theory Division, Physics Department, CERN, \\ CH-1211 Geneva 23, Switzerland \\ ${ }^{c} I I$. Institut für Theoretische Physik, Universität Hamburg, \\ D-22761 Hamburg, Germany \\ ${ }^{d}$ Lorentz Institute for Theoretical Physics, Leiden University, \\ NL-2333 CA Leiden, The Netherlands \\ ${ }^{e}$ Inst. de Th. des Phén. Phys., Ecole Polytechnique Fédérale de Lausanne, \\ CH-1015 Lausanne, Switzerland
}

\begin{abstract}
We develop a method for constructing metastable de Sitter vacua in $\mathcal{N}=1$ supergravity models describing the no-scale volume moduli sector of Calabi-Yau string compactifications. We consider both heterotic and orientifold models. Our main guideline is the necessary condition for the existence of metastable vacua coming from the Goldstino multiplet, which constrains the allowed scalar geometries and supersymmetry-breaking directions. In the simplest non-trivial case where the volume is controlled by two moduli, this condition simplifies and turns out to be fully characterised by the intersection numbers of the Calabi-Yau manifold. We analyse this case in detail and show that once the metastability condition is satisfied it is possible to reconstruct in a systematic way the local form of the superpotential that is needed to stabilise all the fields. We apply then this procedure to construct some examples of models where the superpotential takes a realistic form allowed by flux backgrounds and gaugino condensation effects, for which a viable vacuum arises without the need of invoking corrections to the Kähler potential breaking the noscale property or uplifting terms. We finally discuss the prospects of constructing potentially realistic models along these lines.
\end{abstract}




\section{Introduction}

Current cosmological observations convincingly suggest that our universe is undergoing an accelerated expansion. The simplest model accounting for this result involves backgrounds with a tiny positive cosmological constant. This has lead in the past years to a lot of activity in the search of de Sitter $(\mathrm{dS})$ vacua in the four-dimensional low-energy effective supergravity description of string theory compactifications. It is now well understood that effects like gaugino condensation and background fluxes can induce terms in the effective superpotential that allow to stabilise many or even all of the moduli fields. However, this generically leads to a supersymmetric ground state which is either anti-de Sitter (AdS) or Minkowski space, and it is surprisingly difficult to obtain non-supersymmetric $\mathrm{dS}$ vacua [1-4]. One generic way of overcoming this difficulty is to start from a setting leading to an AdS vacuum and add to it some additional sources of hard supersymmetry breaking, like anti-D3 branes [5] or other localised sources [6,7], to uplift the vacuum energy. However, the addition of such sources does not admit a transparent effective supergravity description, and refinements of this scenario have been considered where the uplifting sector breaks supersymmetry softly and contains additional light degrees of freedom [8-15]. Alternatively, one may achieve dS vacua in a more genuine way thanks to leading perturbative or non-perturbative corrections to the Kähler potential [16-20]. In that case, however, one has to make sure that higher-order subleading corrections are under control.

Despite of the success of the above approaches in producing viable vacua, it would be desirable to have models where metastability is granted from the onset, without the need to incur into either subleading corrections or an additional uplifting sector for help. Ideally, one may want to achieve this within the sector of the moduli fields. The simplest option could be to use just the dilaton, which universally spans the coset space $S U(1,1) / U(1)$, but this has been excluded unless uncontrollably large corrections arise for the geometry [21-23]. Another interesting possibility could be to use only the volume moduli (also called Kähler moduli), which have the universal characteristics of spanning a scalar manifold with a no-scale property. Interestingly, no explicit example is known so far where a viable vacuum is produced without invoking corrections to the Kähler potential breaking its no-scale structure. In the simplest cases where the moduli space is a coset manifold with covariantly-constant curvature, like in the case of one modulus or more generically for $n$ moduli in orbifold limits of Calabi-Yau (CY) compactifications, it has been proved in $[23,24]$ (see also [22]) that dS vacua are in fact unavoidably unstable, because one of the scalar partners of the Goldstino always has a semi-negative mass-squared, for any superpotential. It was however shown later in [25] (see also [26]) that this no-go theorem can be evaded when the moduli span a less constrained space, 
like for smooth CY compactifications. One of the main results deduced in [25], following the line of reasoning of $[23,24]$, is a necessary condition on the Kähler geometry of the moduli space for a metastable dS vacuum to possibly arise. This condition depends on the intersection numbers $d_{i j k}$ and thus restricts the type of CY manifold that can be used. Furthermore, it also constrains the direction in field space along which supersymmetry is allowed to be broken, and thus implicitly restricts the form of the superpotential as well.

The aim of this paper is to analyse in more detail such models, and to study how to determine a superpotential which allows for metastable de Sitter vacua for a given choice of CY manifold. We shall focus on the simplest non-trivial class of models involving two volume moduli, for which the metastability condition simplifies and can be made more explicit, but we believe that the situation for models with more volume moduli should be qualitatively similar. We will then look for a systematic procedure to reconstruct the required form of the superpotential that is needed to achieve stabilisation of all the moduli, once the metastability condition on the Kähler geometry is satisfied.

The paper is organised as follows. In Section 2 we briefly review the results of refs. [2325] regarding the metastability of supersymmetry-breaking vacua and their implications. In Section 3 we apply these results to the more particular case of CY string models with two volume moduli, and deduce which type of models can possibly allow viable vacua. In Section 4 we further analyse those models satisfying the metastability condition, and describe a procedure to determine the type of superpotential that is required to actually get a metastable dS vacuum. In Section 5 we provide explicit examples of string models with a volume moduli sector satisfying all these requirements and admitting a metastable dS vacuum. Finally, in Section 6 we make some concluding remarks.

\section{$2 \quad$ Metastability in supergravity}

Let us start by reviewing the analysis of the stability of non-supersymmetric vacua with non-negative cosmological constant in $\mathcal{N}=1$ supergravity models, following refs. [23, 24] and $[25,26] 11$ We assume here that vector multiplets play a negligible role in the dynamics of supersymmetry breaking and focus thus on theories involving only chiral multiplets 2

Recall first that the most general two-derivative Lagrangian for a supergravity theory with $n$ chiral superfields is entirely determined by the function $G=K+\ln |W|^{2}$, which depends on the chiral superfields $\Phi^{i}$ and their conjugates $\bar{\Phi}^{\bar{\imath}}$ through a real Kähler potential $K$ and a holomorphic superpotential $W$ ? The scalar fields span a Kähler manifold

\footnotetext{
${ }^{1}$ See [27] for a similar analysis in the context of $\mathcal{N}=2$ supergravity with only hypermultiplets.

${ }^{2}$ See [28] for a study of the effects of vector multiplets.

${ }^{3}$ We set $M_{P l}=1$ and denote derivatives with respect to $\phi^{i}$ and $\bar{\phi}^{j}$ by lower indices $i$ and $\bar{\jmath}$.
} 
with a metric given by $g_{i \bar{\jmath}}=K_{i \bar{\jmath}}$, for which the only non-vanishing components of the Christoffel connection and Riemann tensor are $\Gamma_{i j}^{k}=g^{k \bar{l}} K_{i j \bar{l}}$ (and its conjugate), and $R_{i \bar{\jmath} m \bar{n}}=K_{i \bar{\jmath} m \bar{n}}-K_{i m \bar{l}} g^{\bar{l} k} K_{k \bar{\jmath} \bar{n}}$ (and permutations). The chiral auxiliary fields are fixed by their equations of motion to be $F^{i}=m_{3 / 2} G^{i}$, with a scale set by the gravitino mass $m_{3 / 2}=e^{G / 2}$. Whenever $F^{i} \neq 0$ at the vacuum, supersymmetry is spontaneously broken and the direction $G^{i}$ in the space of chiral fermions defines the Goldstino fermion which is absorbed by the gravitino in the process of supersymmetry breaking. We shall describe this direction also in the scalar field space by the unit vector

$$
f_{i}=\frac{G_{i}}{\sqrt{G^{k} G_{k}}} .
$$

Moreover, we will parametrise the cosmological constant in terms of the gravitino mass through the dimensionless quantity

$$
\gamma=\frac{V}{3 m_{3 / 2}^{2}} .
$$

The scalar fields have a kinetic term controlled by the Kähler metric $g_{i \bar{\jmath}}$, which is thus assumed to be positive-definite, and a potential $V$ that takes the following simple form:

$$
V=e^{G}\left(G^{i} G_{i}-3\right) .
$$

Supersymmetry-breaking metastable vacua with non-negative cosmological constant are associated to local minima of the potential at which $G^{i} \neq 0$ and $V \geq 0$. The $n$ complex stationarity condition are derived by computing $V_{i}=\nabla_{i} V$ and read:

$$
V_{i}=e^{G}\left(G_{i}+G^{k} \nabla_{i} G_{k}\right)+G_{i} V=0 .
$$

The $2 n$ dimensional mass matrix for scalar fluctuations around such a vacuum takes the form

$$
M^{2}=\left(\begin{array}{l}
V_{i \bar{\jmath}} V_{i j} \\
V_{\bar{\imath}} V_{\bar{\imath}}
\end{array}\right),
$$

in terms of the second derivatives of the potential $V_{i \bar{\jmath}}=\nabla_{i} \nabla_{\bar{\jmath}} V$ and $V_{i j}=\nabla_{i} \nabla_{j} V$, which can also be computed using covariant derivatives since the extra connection terms vanish by the stationarity conditions, and read:

$$
\begin{aligned}
V_{i \bar{\jmath}} & =e^{G}\left(G_{i \bar{\jmath}}+\nabla_{i} G_{k} \nabla_{\bar{\jmath}} G^{k}-R_{i \bar{\jmath} m \bar{n}} G^{m} G^{\bar{n}}\right)+\left(G_{i \bar{\jmath}}-G_{i} G_{\bar{\jmath}}\right) V, \\
V_{i j} & =e^{G}\left(2 \nabla_{(i} G_{j)}+G^{k} \nabla_{(i} \nabla_{j)} G_{k}\right)+\left(\nabla_{(i} G_{j)}-G_{i} G_{j}\right) V .
\end{aligned}
$$

The metastability condition is then the requirement that the $2 n$-dimensional mass matrix (2.5) should be positive definite. 


\subsection{Necessary condition for metastability}

As discussed in detail in $[25,26]$ it is clear that for a fixed Kähler potential $K$, most of the eigenvalues of $M^{2}$ can be made positive and arbitrarily large by suitably tuning the superpotential $W$. The only restriction comes from the fact that the projection of $V_{i \bar{\jmath}}$ along the Goldstino direction $f^{i}$ is actually constrained by the stationarity conditions (2.4), which imply $\nabla_{i} G_{j} f^{j}=-(1+3 \gamma) f_{i}$, and therefore cannot be adjusted so easily. As a consequence of this fact, in order to study metastability it is sufficient to study the projection of the diagonal block $V_{i \bar{\jmath}}$ of the mass matrix along the Goldstino direction. This projection defines a mass scale $m$ which is related to the masses of the two sGoldstinos and is given by

$$
m^{2} \equiv V_{i \bar{\jmath}} f^{i} f^{\bar{\jmath}} .
$$

A necessary condition for the mass matrix (2.5) to be positive-definite is that $m^{2}>0$. One can then compute this quantity more explicitly and derive a necessary condition for metastability of the vacuum. By using eqs. (2.4) and (2.6), one finds:

$$
m^{2}=\left[3(1+\gamma) \hat{\sigma}\left(f^{i}\right)-2 \gamma\right] m_{3 / 2}^{2},
$$

where包

$$
\hat{\sigma}\left(f^{i}\right) \equiv \frac{2}{3}-R_{i \bar{\jmath} m \bar{n}} f^{i} f^{\bar{\jmath}} f^{m} f^{\bar{n}} .
$$

The condition $m^{2}>0$ implies then the constraint

$$
\hat{\sigma}\left(f^{i}\right)>\frac{2}{3} \frac{\gamma}{1+\gamma} .
$$

Observe that the quantity $R_{i \bar{\jmath} m \bar{n}} f^{i} f^{\bar{\jmath}} f^{m} f^{\bar{n}}$ in eq. (2.10) corresponds to the holomorphic sectional curvature along the Goldstino vector $f^{i}$ and therefore eq. (2.11) is a restriction on the allowed scalar geometries and supersymmetry breaking directions.

Notice that for a fixed $K$ and arbitrary $W$, the direction $f^{i}$ can be varied while keeping the metric and the Riemann tensor fixed. One can then look for the preferred direction $f_{0}^{i}$ that maximises $m^{2}$ with value $m_{0}^{2}$. If $m_{0}^{2}<0$, then one of the sGoldstinos is unavoidably tachyonic, and the vacuum is unstable. If instead $m_{0}^{2}>0$, then the sGoldstinos can be kept non-tachyonic by choosing $W$ such that $f^{i}$ is close-enough to $f_{0}^{i}$. As already mentioned, the rest of the scalars can always be given a positive square mass by further tuning $W$.

\footnotetext{
${ }^{4}$ We use the same notation as in [26] for this quantity, the hat being introduced to distinguish it from the quantity $\sigma$ defined in [25], which has a different normalisation.
} 


\subsection{The sGoldstino mass}

As noted above, $m^{2}$ is related to the square masses of the sGoldstinos, but in general it does not exactly coincide with them, since $f^{i}$ is in general not an eigenvector of the full mass matrix (2.5). We will now show that the preferred direction $f_{0}^{i}$ is instead automatically an eigenvector of the diagonal blocks of (2.5), and the corresponding mass $m_{0}^{2}$ is then more directly related to their mass eigenvalues. More precisely, when the off-diagonal block of (2.5) vanishes one has two degenerate sGoldstinos with square masses given by $m_{0}^{2}$, whereas when the off-diagonal block does not vanish these two masses split.

To prove this statement, let us determine implicitly the direction $f_{0}^{i}$ for which $\mathrm{m}^{2}$ reaches its maximum value $m_{0}^{2}$. To do this, we vary the unit vector $f^{i}$ while keeping the vacuum expectation values (vevs) of the chiral fields fixed, and try to maximise $\hat{\sigma}\left(f^{i}\right)$. Enforcing the constraint $f^{i} f_{i}=1$ with the help of a Lagrange multiplier $\xi$, we are then led to extremise the following functional:

$$
F\left(f^{i}, \xi\right)=\hat{\sigma}\left(f^{i}\right)+\xi\left(g_{i \bar{\jmath}} f^{i} f^{\bar{\jmath}}-1\right) .
$$

Stationarity with respect to $f^{i}$ implies the relation $f_{0 i}=2 \xi_{0}^{-1} R_{i \bar{\jmath} m \bar{n}} f_{0}^{\bar{\jmath}} f_{0}^{m} f_{0}^{\bar{n}}$, which implicitly defines the values of $f_{0}^{i}$ in terms of $\xi_{0}$. Plugging this result back into the constraint $f_{0}^{i} f_{0 i}=1$, which follows from stationarity with respect to $\xi$, determines then $\xi_{0}=2 R_{i \bar{\jmath} m \bar{n}} f_{0}^{i} f_{0}^{\bar{\jmath}} f_{0}^{m} f_{0}^{\bar{n}}$. Putting everything together, one finally finds the following relation implicitly determining $f_{0}^{i}$ :

$$
f_{0 i}=\frac{R_{i \bar{\jmath} m \bar{n}} f_{0}^{\bar{j}} f_{0}^{m} f_{0}^{\bar{n}}}{R_{p \bar{q} r \bar{s}} f_{0}^{p} f_{0}^{\bar{q}} f_{0}^{r} f_{0}^{\bar{s}}} .
$$

Using this relation and the stationarity condition (2.4), one can now easily verify that $f_{0 i}$ is indeed an eigenvector of the matrix $V_{i}^{j}$ with eigenvalue $m_{0}^{2}$ :

$$
V_{i}^{j} f_{0 j}=m_{0}^{2} f_{0 i} .
$$

\section{String models with two moduli}

In this section we will consider more specifically a class of supergravity models arising from the volume moduli sector of CY string compactifications in the low-energy and largevolume limit. We assume that the dilaton and complex structure moduli do not play any relevant role. We will moreover assume that there are only two volume moduli, or that possible additional ones do not play any relevant role either. We will not address in this paper the circumstances under which such a situation can be honestly achieved by making 
the additional moduli heavy and integrating them out 5 Our aim is thus mainly to exhibit the behaviour of a set of two volume moduli with a no-scale Kähler potential.

\subsection{General properties}

Let us start by recalling a few general properties of these types of models, which actually hold true for an arbitrary number of volume moduli. A first important property is that at leading order in the perturbative and low-energy expansions the effective Kähler potential satisfies the no-sale property

$$
K^{i} K_{i}=3
$$

A second property is that $K$ depends only on $\Phi^{i}+\bar{\Phi}^{i}$, i.e. each field enjoys an independent shift symmetry, under which $\delta \phi^{i}=i \lambda$. This allows to drop any distinction between holomorphic and antiholomorphic indices in quantities deduced from $K$. Actually, it turns out that there exists a special coordinate frame in which $e^{-K}$ is a homogeneous function of degree 3 in the fields $\Phi^{i}+\bar{\Phi}^{i}$. One then has:

$$
-\left(\Phi^{i}+\bar{\Phi}^{i}\right) K_{i}=3
$$

Taking a derivative of this relation it then also follows that $K^{i}=-\left(\Phi^{i}+\bar{\Phi}^{i}\right)$. This equation, together with (3.2), implies the no-scale property (3.1), and is thus stronger than it.

In the light of the above properties, it proves convenient to introduce the unit vector defined by the derivatives of the Kähler potential:

$$
k_{i}=\frac{1}{\sqrt{3}} K_{i}
$$

It was shown in [25] that as a result of the no-scale property the function $\hat{\sigma}$ controlling the mass $m^{2}$ vanishes along this direction, for any value of the fields:

$$
\hat{\sigma}\left(k^{i}\right)=0
$$

As thoroughly discussed in [25], this result allows to study the metastability condition by analysing the behaviour of $\hat{\sigma}\left(f^{i}\right)$ in the vicinity of $f^{i}=k^{i}$. In this analysis, a special role is played by the subspace orthogonal to $k^{i}$, which is spanned by a basis of $n-1$ complex unit vectors orthogonal to $k^{i}$.

\footnotetext{
${ }^{5}$ See refs. [29-32] for work in this direction.
} 


\subsection{Models with two moduli}

The general problem of determining whether a dS vacuum may arise in the models under consideration is still quite complicated, even in the light of the restrictions (3.1), (3.2) and (3.4). However, one can fully characterise the metastability condition for two-moduli models. In this case, the field space is of complex dimension 2 and can be conveniently parametrised with a basis of two unit vectors: $k^{i}$ and a vector $n^{i}$ perpendicular to it:

$$
k^{i} n_{i}=0 .
$$

This condition defines $n^{i}$ uniquely, up to an overall phase, in terms of the components of $k^{i}$ and the elements of the metric and its inverse. Denoting by det $g$ the determinant of the metric, one easily finds:

$$
\left(n_{1}, n_{2}\right)=\sqrt{\operatorname{det} g}\left(k^{2},-k^{1}\right), \quad\left(n^{1}, n^{2}\right)=\frac{1}{\sqrt{\operatorname{det} g}}\left(k_{2},-k_{1}\right) .
$$

Since the space perpendicular to $k^{i}$ is one-dimensional, it coincides with the space parallel to $n^{i}$, and the projection operator $P^{i j}$ onto such a subspace is simply given by

$$
P^{i j}=g^{i j}-k^{i} k^{j}=n^{i} n^{j}
$$

We may now decompose the unit vector $f^{i}$ defining the Goldstino direction in terms of the two orthogonal vectors $n^{i}$ and $k^{i}$. Up to an overall phase, that we shall not display explicitly, we can parametrise the result in terms of an angle $\chi$ and a relative phase $\delta$, and write:

$$
\begin{array}{ll}
f^{i}=\sin \chi k^{i}+e^{i \delta} \cos \chi n^{i}, & f_{i}=\sin \chi k_{i}+e^{-i \delta} \cos \chi n_{i}, \\
f^{\bar{\imath}}=\sin \chi k^{i}+e^{-i \delta} \cos \chi n^{i}, & f_{\bar{\imath}}=\sin \chi k_{i}+e^{i \delta} \cos \chi n_{i} .
\end{array}
$$

To proceed further and be more explicit, we need now to distinguish between the two classes of heterotic and orientifold models. In ref. [25] it was found that in both cases the possibility of achieving a metastable dS vacuum is linked to the sign of the discriminant $\Delta$ of the cubic polynomial defined by the intersection numbers $d_{i j k}$, after scaling out one variable, and reads

$$
\Delta=-27\left(d_{111}^{2} d_{222}^{2}-3 d_{112}^{2} d_{122}^{2}+4 d_{111} d_{122}^{3}+4 d_{112}^{3} d_{222}-6 d_{111} d_{112} d_{122} d_{222}\right) .
$$

If $\Delta<0$ the heterotic version can potentially admit dS vacua but not the orientifold one. Viceversa, if $\Delta>0$ the orientifold version can but the heterotic cannot. In what follows we compute $\hat{\sigma}$ explicitly in terms of $\chi$ and $\delta$ parameterising $f^{i}$ for both of these cases. 


\subsection{Heterotic models}

In heterotic models, the effective Kähler potential takes the following simple form in the large volume limit:6

$$
K=-\log \mathcal{V}, \quad \mathcal{V}=\frac{4}{3} d_{i j k} t^{i} t^{j} t^{k}
$$

In this expression, $d_{i j k}$ denotes the intersection numbers of the CY manifold and $t^{i}$ are the volume moduli. In this case, the $t^{i}$ can be promoted in a simple way to (scalar components of) chiral superfields, by setting $t^{i}=\left(T^{i}+\bar{T}^{i}\right) / 2$.

From the form of the Kähler potential (3.10) it follows that $K^{i}=-\left(T^{i}+\bar{T}^{i}\right.$ ) and $K_{i}=-1 / 2 e^{K} d_{i m n} K^{m} K^{n}$. The metric and the Riemann tensor are then given by (see [25] for more details)

$$
\begin{aligned}
g_{i j} & =e^{K} d_{i j n} K^{n}+K_{i} K_{j}, \\
R_{i j m n} & =g_{i j} g_{m n}+g_{i n} g_{m j}-e^{2 K} d_{i m p} g^{p q} d_{q j n} .
\end{aligned}
$$

Using this expression, as well as (3.8) it is then possible to rewrite $\hat{\sigma}\left(f^{i}\right)$ in the form $\hat{\sigma}_{\mathcal{H}}\left(f^{i}\right)=-2 \hat{s}^{i} \hat{s}_{i}+\hat{\omega}$, where

$$
\begin{aligned}
& \hat{s}^{i}=n^{i}\left[\frac{2}{\sqrt{3}} \tan \chi \cos \delta-\frac{1}{2} e^{K} d_{p q r} n^{p} n^{q} n^{r}\right] \cos ^{2} \chi, \\
& \hat{\omega}=\left[\frac{3}{2}\left(e^{K} d_{p q r} n^{p} n^{q} n^{r}\right)^{2}-1\right] \cos ^{4} \chi .
\end{aligned}
$$

On the other hand, it was shown in [25] that

$$
\frac{3}{2}\left(e^{K} d_{p q r} n^{p} n^{q} n^{r}\right)^{2}-1=a_{\mathcal{H}},
$$

where

$$
a_{\mathcal{H}} \equiv-\frac{\Delta}{24} \frac{e^{4 K}}{(\operatorname{det} g)^{3}} \geq-1
$$

Putting all of these results back into eqs. (3.13)-(3.14), and introducing the sign $s_{\mathcal{H}}=$ $\operatorname{sign}\left(d_{p q r} n^{p} n^{q} n^{r}\right)$, we finally obtain

$$
\hat{\sigma}(\chi, \delta)=\left[a_{\mathcal{H}}-\frac{8}{3}\left(\tan \chi \cos \delta-s_{\mathcal{H}} \sqrt{\frac{1+a_{\mathcal{H}}}{8}}\right)^{2}\right] \cos ^{4} \chi .
$$

\footnotetext{
${ }^{6}$ The discussion of this section is also valid for certain classes of orientifold compactifications where the Kähler potential exhibits the same form (3.10). An example of this are compactifications of type IIB with O5/O9-orientifold planes [33].
} 
Observe that $\hat{\sigma}$ depends on the vevs of moduli only through the quantity $a_{\mathcal{H}} \cdot 7$ Notice also that the squared term can always be set to zero by tuning $\chi$. On the other hand, as long as $\Delta<0$ the term proportional to $a_{\mathcal{H}}$ is always positive. For a fixed value of $a_{\mathcal{H}} \in[0,+\infty)$, we may then compute the maximal value $\hat{\sigma}_{0}$ that can be achieved for $\hat{\sigma}$. This corresponds to finding the optimal direction $f_{0}^{i}$ discussed in Section 2.2 , The relevant extremum occurs at

$$
\delta_{0}=0, \quad \tan \chi_{0}=s_{\mathcal{H}} \sqrt{\frac{1+a_{\mathcal{H}}}{8}}(1+\epsilon)
$$

where $\epsilon$ is a quantity still to be determined. One has then

$$
\hat{\sigma}_{0}=\frac{64\left[a_{\mathcal{H}}-\left(1+a_{\mathcal{H}}\right) \epsilon^{2} / 3\right]}{\left[8+\left(1+a_{\mathcal{H}}\right)(1+\epsilon)^{2}\right]^{2}} .
$$

Notice first that one gets a lower bound on the size that $\hat{\sigma}$ can reach by setting $\epsilon \simeq 0$, which corresponds to setting to zero the negative definite part of the numerator. This is what was done in [26], and results in the value $\hat{\sigma}_{0} \simeq 64 a_{\mathcal{H}} /\left(9+a_{\mathcal{H}}\right)^{2}$. This expression has an extremum at $a_{\mathcal{H}}=9$ where it reaches its maximal value $\hat{\sigma}_{0} \simeq 16 / 9$. The true maximal value $\hat{\sigma}_{0}$ is however obtained for a non-vanishing value of $\epsilon$ determined by the stationarity condition $\partial \hat{\sigma} / \partial \epsilon=0$, which is a cubic polynomial. This polynomial accidentally factorises in a simple way in this case, and it is actually possible to find the following simple expression for the value of $\epsilon$ :

$$
\epsilon=\frac{3}{2}\left(\frac{\sqrt{1+a_{\mathcal{H}} / 9}}{\sqrt{1+a_{\mathcal{H}}}}-1\right)
$$

Notice that $\epsilon$ is only small for small $a_{\mathcal{H}}$. This means that the exact $\hat{\sigma}_{0}$ will depart significantly from the approximate one for large values of $a_{\mathcal{H}}$. Plugging (3.20) back into (3.19) one finds that this is given by:

$$
\hat{\sigma}_{0}=\frac{128}{3} \frac{a_{\mathcal{H}}+9 \sqrt{\left(1+a_{\mathcal{H}}\right)\left(1+a_{\mathcal{H}} / 9\right)}-9}{\left(21+a_{\mathcal{H}}-3 \sqrt{\left(1+a_{\mathcal{H}}\right)\left(1+a_{\mathcal{H}} / 9\right)}\right)^{2}},
$$

From eq. (3.21) we see that $\hat{\sigma}_{0}$ grows asymptotically as $2 / 3 a_{\mathcal{H}}$ for large values of $a_{\mathcal{H}}$ and can thus be made arbitrarily large and positive. This means that for heterotic models the sGoldstino mass scale $m$ can be made arbitrarily large by tuning the value of the moduli. As we shall see in the following subsection, this is not the case for orientifold models with two moduli.

\footnotetext{
${ }^{7}$ Certainly, for a given choice of the superpotential, $\chi$ and $\delta$ also depend on the moduli. Nevertheless, in the present approach $\chi$ and $\delta$ are independent of the moduli in the sense that we are leaving free the parameters entering the superpotential that a posteriori will do the job of stabilising the moduli. How to determine these parameters will be the subject of Section 4
} 


\subsection{Orientifold models}

Let us consider now the case of orientifold models. We focus on type IIB models with O3/O7 planes, where the effective Kähler potential in the large-volume limit takes the form [33]

$$
K=-2 \log \mathcal{V}, \quad \mathcal{V}=\frac{1}{48} d^{i j k} v_{i} v_{j} v_{k}
$$

In this expression $d^{i j k}$ denotes the collection of intersection numbers of the CY (rescaled by a factor of $1 / 8$ for convenience) and $v_{i}$ are the volume moduli. However, the $v^{i}$ do not directly correspond to the real part of scalar components of chiral superfields in this case. These are instead given by new fields $\rho^{i}$, related to the $v_{i}$ via the quadratic relation

$$
\rho^{i}=\frac{\partial \mathcal{V}}{\partial v_{i}}=\frac{1}{16} d^{i j k} v_{j} v_{k}
$$

One then has to invert this relation and express the $v_{i}$ in terms of the $\rho^{i}$. After that, one obtains the superfield dependence of $K$ by setting $\rho^{i}=\left(T^{i}+\bar{T}^{i}\right) / 2$. In general, this can however not be given explicitly and the Kähler potential (3.22) remains an implicit function of the $T^{i}$. Note finally that we have used lower indices for the fields $v_{i}$ in order to get upper indices for the fields $\rho^{i}$. Correspondingly we have used upper indices for the intersection numbers $d^{i j k}$, but it should be stressed that they are the same objects as in the heterotic case.

From the above implicit definition of the Kähler potential it follows that $K_{i}=-\frac{1}{2} e^{K / 2} v_{i}$ and $K^{i}=-\left(T^{i}+\bar{T}^{i}\right)$. The metric and the Riemann tensor are then found to be (see [25] and [34] for more details):

$$
\begin{aligned}
g_{i j}= & K_{i} K_{j}+e^{-K} \hat{d}_{i j k} K^{k} \\
R_{i j m n}= & -g_{i m} g_{j n}+e^{-2 K}\left(\hat{d}_{i j k} g^{k l} \hat{d}_{l m n}+\hat{d}_{i n k} g^{k l} \hat{d}_{l j m}\right)+g_{i n} K_{j} K_{m}+g_{j m} K_{i} K_{n} \\
& +g_{i m} K_{j} K_{n}+g_{j n} K_{i} K_{m}+g_{i j} K_{m} K_{n}+g_{m n} K_{i} K_{j}-3 K_{i} K_{j} K_{m} K_{n} \\
& -e^{-K}\left(\hat{d}_{i m j} K_{n}+\hat{d}_{i m n} K_{j}+\hat{d}_{i n j} K_{m}+\hat{d}_{n m j} K_{i}\right),
\end{aligned}
$$

where we introduced the notation

$$
\hat{d}_{i j k} \equiv g_{i p} g_{j q} g_{k l} d^{p q l}
$$

Inserting these expressions into the definition of $\hat{\sigma}\left(f^{i}\right)$ in (2.10) and using the parametrisation (3.8) for $f^{i}$ we can as before rewrite $\hat{\sigma}\left(f^{i}\right)$ in the form $\hat{\sigma}\left(f^{i}\right)=-2 \hat{s}^{i} \hat{s}_{i}+\hat{\omega}$ where:

$$
\begin{aligned}
& \hat{s}^{i}=n^{i}\left[\frac{2}{\sqrt{3}} \tan \chi \cos \delta-\frac{1}{2} e^{-K} d^{p q r} n_{p} n_{q} n_{r}\right] \cos ^{2} \chi, \\
& \hat{\omega}=\left[1-\frac{3}{2}\left(e^{-K} d^{p q r} n_{p} n_{q} n_{r}\right)^{2}\right] \cos ^{4} \chi .
\end{aligned}
$$


On the other hand, it can be shown that [25]

$$
1-\frac{3}{2}\left(e^{-K} d^{p q r} n_{p} n_{q} n_{r}\right)^{2}=a_{\mathcal{O}}
$$

where

$$
a_{\mathcal{O}} \equiv \frac{\Delta}{24} \frac{(\operatorname{det} g)^{3}}{e^{4 K}} \leq 1
$$

Putting all of this together, and introducing the $\operatorname{sign} s_{\mathcal{O}}=\operatorname{sign}\left(d^{p q r} n_{p} n_{q} n_{r}\right)$, we finally obtain

$$
\hat{\sigma}(\chi, \delta)=\left[a_{\mathcal{O}}-\frac{8}{3}\left(\tan \chi \cos \delta-s_{\mathcal{O}} \sqrt{\frac{1-a_{\mathcal{O}}}{8}}\right)^{2}\right] \cos ^{4} \chi .
$$

It is clear that, as before, the squared term can always be set to zero by tuning $\chi$ and then $\hat{\sigma}>0$ as long as the term proportional to $a_{\mathcal{H}}$ is positive, which is the case when $\Delta>0$.

As in the previous subsection, we can now ask what is the maximum value for $\hat{\sigma}$ obtained by varying the Goldstino direction $f^{i}$, for a given $a_{\mathcal{O}} \in[0,1]$. The relevant extremum occurs for

$$
\delta_{0}=0, \quad \tan \chi_{0}=s_{\mathcal{O}} \sqrt{\frac{1-a_{\mathcal{O}}}{8}}(1+\epsilon) .
$$

One then has

$$
\hat{\sigma}_{0}=\frac{64\left[a_{\mathcal{O}}-\left(1-a_{\mathcal{O}}\right) \epsilon^{2} / 3\right]}{\left[8+\left(1-a_{\mathcal{O}}\right)(1+\epsilon)^{2}\right]^{2}} .
$$

One gets as before a lower bound on $\hat{\sigma}_{0}$ by setting $\epsilon \simeq 0$. This gives the approximate value $\hat{\sigma}_{0} \simeq 64 a_{\mathcal{O}} /\left(9-a_{\mathcal{O}}\right)^{2}$, which grows as $a_{\mathcal{O}}$ is increased until the point $a_{\mathcal{O}}=1$, where it reaches its maximal value $\hat{\sigma}_{0} \simeq 1$. But again the exact maximal value of $\hat{\sigma}$ for a given $a_{\mathcal{O}}$ is larger and occurs for a in general non-vanishing value of $\epsilon$ determined by the condition $\partial \hat{\sigma} / \partial \epsilon=0$, which is again a cubic polynomial. In this case, this polynomial is generic, and the expression for the value of $\epsilon$ is somewhat complicated. One finds:

$$
\epsilon=\frac{\sqrt{1+5 a_{\mathcal{O}} / 9}}{\sqrt{1-a_{\mathcal{O}}}}(3 \sin \theta-\sqrt{3} \cos \theta)
$$

where

$$
\theta \equiv \frac{1}{3} \arccos \left(\frac{a_{\mathcal{O}}}{\sqrt{3}} \frac{\sqrt{1-a_{\mathcal{O}}}}{\left(1+5 a_{\mathcal{O}} / 9\right)^{3 / 2}}\right) .
$$

Plugging this back into (3.33), one finds that the exact maximal value $\hat{\sigma}_{0}$ is given by a relatively complicated expression, which we do not report here. Fortunately, one can however check that the quantity $\epsilon$ given by (3.34) is always quite small for any value of 
$a_{\mathcal{O}} \in[0,1]$. In particular, one easily verifies that also the exact $\hat{\sigma}_{0}$ increases monotonically as a function of $a_{\mathcal{O}}$, and that for $a_{\mathcal{O}}=1$ one obtains $\hat{\sigma}_{0}=1$. In practise one can then approximate the maximal value of $\hat{\sigma}$ with the one associated with $\epsilon \simeq 0$, namely

$$
\hat{\sigma}_{0} \simeq \frac{64 a_{\mathcal{O}}}{\left(9-a_{\mathcal{O}}\right)^{2}}
$$

Notice finally that the fact that $\hat{\sigma}$ can be at most 1 implies the following upper bound for the sGoldstino mass scale $m$ :

$$
m^{2} \leq(3+\gamma) m_{3 / 2}^{2}
$$

This is an interesting result concerning the phenomenology of orientifold compactifications. It asserts that the lightest modulus cannot be much heavier than the gravitino. It seems therefore to point towards a large gravitino mass as the only way to ease the cosmological moduli problem [35]. As we shall see during the next section, one can actually saturate the above bound by suitably tuning the superpotential.

\section{Constructing de Sitter vacua with two moduli}

Let us now come to the main point of this paper, namely to the question of how for a given Kähler potential, satisfying the necessary condition for metastability on the sign of $\Delta$, one may construct superpotentials that indeed allow for local minima of the scalar potential $V$ with a non-negative cosmological constant. Our strategy will be to assume some reference values for the fields at the location of the minimum, $T^{1,2}=T_{0}^{1,2}$, and then to reconstruct the local behaviour that $W$ needs to have at that point 8 We will thus consider an expansion of the form:

$$
\begin{aligned}
W(T)= & W_{0}+W_{i}\left(T-T_{0}\right)^{i}+\frac{1}{2} W_{i j}\left(T-T_{0}\right)^{i}\left(T-T_{0}\right)^{j} \\
& +\frac{1}{6} W_{i j k}\left(T-T_{0}\right)^{i}\left(T-T_{0}\right)^{j}\left(T-T_{0}\right)^{k}+\cdots
\end{aligned}
$$

The goal is to determine suitable coefficients $W_{0}, W_{i}, W_{i j}$ and $W_{i j k}$. Higher order terms in the expansion do not affect the masses of scalar fluctuations around the vacuum and can therefore be omitted. Since we are demanding stabilisation at $T^{1,2}=T_{0}^{1,2}$, these coefficients depend on $T_{0}^{1,2}$ via $K$ and its derivatives evaluated at these field values. More precisely, they depend only on $\operatorname{Re} T_{0}^{1,2}$, because of the shift symmetry of $K$. Hence, the vevs of the axions $\operatorname{Im} T^{i}$ do not affect the coefficients in eq. (4.1) and can be chosen freely.

\footnotetext{
${ }^{8}$ One may also try to brutally scan over the parameter space of some plausible superpotential for those models that satisfy the metastability necessary condition. However, this proves to be very cumbersome as soon as there are several parameters. In this framework, the algebraic method for finding dS minima developed in ref. [36] may perhaps be useful.
} 
Let us now describe a systematic procedure to reconstruct the coefficients $W_{0}, W_{i}, W_{i j}$ and $W_{i j k}$. Notice, before starting, that the freedom in choosing the two vevs $T_{0}^{1,2}$ can be used to achieve any desired value for the volume $\mathcal{V}$, and a suitable positive value for the parameter $a$. More precisely, the value of $a$ fixes the ratio of $T_{0}^{1}$ and $T_{0}^{2}$, whereas the value of the volume $\mathcal{V}$ fixes their overall size. Note also from eq. (4.1) that rescaling the vevs of the fields $T_{0}^{1,2}$ can be compensated by rescaling the coefficients appropriately, after factorising out the overall superpotential scale $W_{0}$.

\subsection{Tuning $W_{0}$}

The coefficient $W_{0}$ is fixed, modulo a phase that we shall discard, by the value one desires to achieve for the gravitino mass compared to the volume. From the definition of $m_{3 / 2}$ one gets the relation

$$
\left|W_{0}\right|=m_{3 / 2} e^{-K / 2} .
$$

Note that due to the different definitions of the volume $\mathcal{V}$ for heterotic and orientifold models, this equation translates into different relations between $m_{3 / 2}$ and $\mathcal{V}$ in heterotic and orientifold models. In the two cases one finds respectively

$$
\left|W_{0}\right|=m_{3 / 2} \mathcal{V}_{\mathcal{H}}^{1 / 2}, \quad\left|W_{0}\right|=m_{3 / 2} \mathcal{V}_{\mathcal{O}}
$$

In any case, the value of $W_{0}$ fixes the overall scale of the potential.

\section{$4.2 \quad$ Tuning $W_{i}$}

The two coefficients $W_{i}$ are fixed by the value of the cosmological constant and the direction of supersymmetry breaking that one desires to achieve. Indeed, one has by definition $G_{i}=K_{i}+W_{i} / W_{0}$, and $G_{i}$ can be parametrised in terms of $\gamma$ and $f_{i}$ as $G_{i}=\sqrt{3(1+\gamma)} f_{i}$. Recalling also the definition $K_{i}=\sqrt{3} k_{i}$, it follows then that:

$$
\frac{W_{i}}{W_{0}}=\sqrt{3}\left(\sqrt{1+\gamma} f_{i}-k_{i}\right)
$$

This fixes $W_{i} / W_{0}$ in terms of $\gamma$ and $f_{i}$. The direction $f_{i}$, which we have parametrised by $\chi$ and $\delta$ in eq. (3.8), must be chosen inside a cone sufficiently close to the optimal direction $f_{0 i}$, in such a way that $m^{2}>0.9$

\footnotetext{
${ }^{9}$ Note that in eq. (4.4) the overall phase discarded in the parametrisation (3.8) becomes relevant and represents an additional parameter that one can tune.
} 


\subsection{Tuning $W_{i j}$}

The three coefficients $W_{i j}$ are fixed by demanding stationarity of the potential, $\nabla_{i} V=0$, and positivity of the two-dimensional diagonal blocks $V_{i \bar{\jmath}}$ of the mass matrix, which is necessary for positivity of the full mass matrix. It is convenient to first implement the stationarity conditions (2.4). This implies the following two relations, which allow to fix two of the three parameters $W_{i j}$ in terms of the last one (understanding now $G_{i}$ as fixed):

$$
\frac{W_{i j}}{W_{0}} G^{j}=-(1+3 \gamma) G_{i}-G_{\bar{\imath}}+\Gamma_{i j}^{k} G_{k} G^{j}+\frac{W_{i} W_{j}}{W_{0}^{2}} G^{j} .
$$

The remaining parameter among the $W_{i j}$ which is still free is then fixed by demanding positivity of the two-dimensional matrix $V_{i \bar{j}}$. This amounts to requiring that its two eigenvalues are positive. Notice that we have already ensured the positivity of the projection $m^{2}=V_{i \bar{\jmath}} f^{i} f^{\bar{\jmath}}$. Thus, it makes sense now to study the projection of $V_{i \bar{\jmath}}$ along the remaining direction $u^{i}$ orthogonal to $f^{i}$ in order to understand when the positivity of the whole matrix $V_{i \bar{\jmath}}$ is possible. This direction is completely fixed, again modulo an overall phase that we do not display, and is given by:

$$
\begin{array}{ll}
u^{i}=\cos \chi k^{i}-e^{i \delta} \sin \chi n^{i}, & u_{i}=\cos \chi k_{i}-e^{-i \delta} \sin \chi n_{i}, \\
u^{\bar{\imath}}=\cos \chi k^{i}-e^{-i \delta} \sin \chi n^{i}, & u_{\bar{\imath}}=\cos \chi k_{i}-e^{i \delta} \sin \chi n_{i} .
\end{array}
$$

We are then led to compute

$$
m^{\prime 2} \equiv V_{i \bar{\jmath}} u^{i} u^{\bar{\jmath}}
$$

Using the fact that $\nabla_{i} G_{j} u^{i} f^{j}=0$ by the stationarity condition, one finds that this second mass scale is given by:

$$
m^{\prime 2}=\left[1+3 \gamma-3(1+\gamma) \hat{\beta}\left(u^{i}\right)+\left|\nabla_{i} G_{j} u^{i} u^{j}\right|^{2}\right] m_{3 / 2}^{2},
$$

where

$$
\hat{\beta}\left(u^{i}\right)=R_{i j m n} u^{i} u^{\bar{\jmath}} f^{m} f^{\bar{n}} .
$$

From eq. (4.8) we see that it is always possible to tune the quantity $\nabla_{i} G_{j}$ in order to make the last positive term dominate and achieve $m^{\prime 2}>0$, compatibly with the two stationarity conditions that also involve $\nabla_{i} G_{j}$, since there are three parameters $W_{i j}$. On the other hand, the matrix $V_{i \bar{\jmath}}$ has in general a non-zero mixing between the $f^{i}$ and $u^{i}$ directions, which is given by

$$
V_{i \bar{\jmath}} u^{i} f^{\bar{\jmath}}=-3(1+\gamma) m_{3 / 2}^{2} R_{i j m n} u^{i} f^{\bar{\jmath}} f^{m} f^{\bar{n}} .
$$

Since this quantity is independent of $\nabla_{i} G_{j}$, it is now evident that it is always possible to tune the value of $m^{\prime 2}$ until both eigenvalues of $V_{i \bar{\jmath}}$ become positive. 
A simple although not mandatory possibility to fix unambiguously the free parameter left among the $W_{i j}$ after imposing the stationarity condition is to require that $f_{i}$ should be aligned along the optimal direction $f_{0}^{i}$ maximising $m^{2}$. In that case the orthogonal direction $u_{i}$ is then also fixed to some $u_{0}^{i}$. In this situation, eq. (2.13) implies that one has $V_{i \bar{\jmath}} u_{0}^{i} f_{0}^{\bar{\jmath}}=0$, so that $m^{2}$ and $m^{\prime 2}$ coincide with the two eigenvalues of $V_{i \bar{\jmath}}$. Additionally, the quantity $\hat{\beta}$ takes a definite value, which is different for heterotic and orientifold models and depends on $a_{\mathcal{H}}$ and $a_{\mathcal{O}}$ respectively. After a straightforward but lengthy computation one finds:

$$
\begin{aligned}
& \hat{\beta}_{\mathcal{H} 0}=\frac{1}{24}\left(9-2 a_{\mathcal{H}}+\left(7+2 a_{\mathcal{H}}\right) \cos 4 \chi_{0}+4 s_{\mathcal{H}} \sqrt{2\left(1+a_{\mathcal{H}}\right)} \sin 4 \chi_{0}\right), \\
& \hat{\beta}_{\mathcal{O} 0}=\frac{1}{24}\left(9-4 a_{\mathcal{O}}+\left(7+4 a_{\mathcal{O}}\right) \cos 4 \chi_{0}+4 s_{\mathcal{O}} \sqrt{2\left(1-a_{\mathcal{O}}\right)} \sin 4 \chi_{0}\right) .
\end{aligned}
$$

In these expressions, the quantity $\chi_{0}$ is the one that leads to the maximal value $\hat{\sigma}_{0}$ for $\hat{\sigma}$, namely $\tan \chi_{0}=s \sqrt{(1 \pm a) / 8}(1+\epsilon)$. For heterotic models, one has to use the exact value (3.20), but for orientifold it is good enough to use the approximate value $\epsilon \simeq 0$. In this way one finds:

$$
\begin{aligned}
& \hat{\beta}_{\mathcal{H} 0}=\frac{9-a_{\mathcal{H}}+9 \sqrt{\left(1+a_{\mathcal{H}}\right)\left(1+a_{\mathcal{H}} / 9\right)}}{27+2 a_{\mathcal{H}}}, \\
& \hat{\beta}_{\mathcal{O} 0} \simeq \frac{2}{3}\left(1-12 \frac{a_{\mathcal{O}}\left(1-a_{\mathcal{O}}\right)}{\left(9-a_{\mathcal{O}}\right)^{2}}\right) .
\end{aligned}
$$

We see in particular that both quantities remain bounded respectively by 1 and $2 / 3$ in the allowed ranges for $a$.

\subsection{Tuning $W_{i j k}$}

Finally, the four coefficients $W_{i j k}$ need to be chosen in such a way that all of the four eigenvalues of the full mass matrix $M^{2}$ are positive, even after taking into account the effect of the off-diagonal block $V_{i j}$. Solving then the expression for $V_{i j}$ in terms of the $W_{i j k}$, one deduces the following three relations (where now both $G_{i}$ and $\nabla_{i} G_{j}$ are understood as fixed):

$$
\begin{aligned}
\frac{W_{i j k}}{W_{0}} G^{k}= & {\left[R_{i j k m} G^{\bar{m}}+\Gamma_{i j}^{m} \nabla_{m} G_{k}+\Gamma_{(i k}^{m} \nabla_{m} G_{j)}-2 \frac{W_{i} W_{j} W_{k}}{W_{0}^{3}}+2 \frac{W_{(i} W_{j) k}}{W_{0}^{2}}+\frac{W_{k} W_{i j}}{W_{0}^{2}}\right.} \\
& \left.+\Gamma_{(i k}^{m}\left(\frac{W_{m j)}}{W_{0}}-\frac{W_{m} W_{j)}}{W_{0}^{2}}\right)\right] G^{k}-(2+3 \gamma) \nabla_{(i} G_{j)}+3 \gamma G_{i} G_{j}+\frac{V_{i j}}{m_{3 / 2}^{2}} .
\end{aligned}
$$

Recall that for $V_{i j}=0$ the mass spectrum is degenerate, with two states for each of the two eigenvalues of $V_{i \bar{\jmath}}$, which have already been adjusted to be positive with the previous 
step. When instead $V_{i j} \neq 0$, the spectrum splits and one has to make sure that no eigenvalue becomes negative. This represents three constraints on the four parameters $W_{i j k}$. If for simplicity one requires $V_{i j}=0$, then these become three relations, which allow to express three of the four parameters $W_{i j k}$ in terms of the last one. More generally, we can leave $V_{i j}$ arbitrary and compute the four eigenvalues as functions of the $W_{i j k}$ 's. In generic situations it is hard to do this in an analytic way, but it can be easily done with computer assistance. One can then scan this multi-parameter space for regions where all masses are positive.

The next step is to match these 'local superpotentials' with the expansion of some stringmotivated superpotential around the given vevs. To this end we will consider in the next section superpotentials with enough parameters and determine these parameters in such a way that the Taylor expansion around the extremum matches the cubic superpotential constructed as outlined above.

\section{Examples of models with dS vacua}

Let us now apply the procedure described in last section to construct some illustrative examples of string models with a sector of two volume moduli admitting a metastable dS vacuum. For simplicity, we shall focus on the case where the cosmological constant vanishes $(\gamma=0)$ and on separable superpotentials of the form $W\left(T^{1}, T^{2}\right)=W^{(1)}\left(T^{1}\right)+W^{(2)}\left(T^{2}\right)$. This choice implies further restrictions on the coefficients of the Taylor expansion of the superpotential about the vacuum, namely $W_{12}=W_{112}=W_{221}=0$, and the existence of a solution with these characteristics is no longer guaranteed from the beginning. We will however see that it is nevertheless possible to find simple examples of this type.

\subsection{Orientifold models}

Let us start with orientifold models. For these models, the way in which the dilaton and the complex structure moduli may be stabilised is well understood [1], and restricting to the sector of volume moduli may be justified. In this case, the necessary condition for metastability is that the discriminant $\Delta$ should be positive. As a prototype example, let us take a CY manifold with intersection numbers given by $d^{111}=-1, d^{112}=0, d^{122}=1$ and $d^{222}=0$, for which $\Delta=108>0$. The Kähler potential is then found to take the 
following form:

$$
\begin{aligned}
K=-\log & {\left[\frac{8}{9}\left(\left(T^{1}+\bar{T}^{1}\right)+\sqrt{\left(T^{1}+\bar{T}^{1}\right)^{2}+\left(T^{2}+\bar{T}^{2}\right)^{2}}\right)\right.} \\
& \left.\left(\frac{\left(T^{2}+\bar{T}^{2}\right)^{2}+\left(T^{1}+\bar{T}^{1}\right)^{2}-\left(T^{1}+\bar{T}^{1}\right) \sqrt{\left(T^{1}+\bar{T}^{1}\right)^{2}+\left(T^{2}+\bar{T}^{2}\right)^{2}}}{T^{2}+\bar{T}^{2}}\right)^{2}\right] .
\end{aligned}
$$

We require that at the stationary point one should have $a_{\mathcal{O}}=1$. As seen in Section 3.4, this choice allows to maximise the sGoldstino mass and corresponds to setting $\hat{s}^{i}=0$. We will moreover require that the volume takes some definite numerical value $\mathcal{V}_{\mathcal{O}}$. These two conditions fix the vevs of the two fields to the following values, in units of $\mathcal{V}_{\mathcal{O}}^{2 / 3}$ :

$$
\begin{array}{|l|l|}
\hline T_{0}^{1} & 0.412741 \\
T_{0}^{2} & 0.714888 \\
\hline
\end{array}
$$

Applying then the procedure described in previous section, in such a way to achieve some definite numerical value $m_{3 / 2}$ for the gravitino mass, we find that the local behaviour that the superpotential needs to have is specified by the following Taylor coefficients, in units of $m_{3 / 2} \mathcal{V}_{\mathcal{O}}$ for $W_{0}, m_{3 / 2} \mathcal{V}_{\mathcal{O}}^{1 / 3}$ for $W_{i}, m_{3 / 2} \mathcal{V}_{\mathcal{O}}^{-1 / 3}$ for $W_{i i}$ and $m_{3 / 2} \mathcal{V}_{\mathcal{O}}^{-1}$ for $W_{i i i}$ :

\begin{tabular}{|l|r|}
\hline$W_{0}$ & 1.000000 \\
$W_{1}$ & 2.021311 \\
$W_{2}$ & 0.931223 \\
$W_{11}$ & 0.999657 \\
$W_{22}$ & -0.797685 \\
$W_{111}$ & -0.827204 \\
$W_{222}$ & 3.308820 \\
\hline
\end{tabular}

In this way, the four physical square-mass eigenvalues $m_{i}^{2}$ at the minimum, obtained after canonically normalising the fields, are given by $2.77,2.95,3.86,5.14$ in units of $m_{3 / 2}^{2}$.

Notice that the coefficients (5.3) scale in the following way with the size $T_{0} \sim \mathcal{V}_{\mathcal{O}}^{2 / 3}$ of the field vevs:

$$
W_{0}: W_{i}: W_{i i}: W_{i i i} \sim 1: T_{0}^{-1}: T_{0}^{-2}: T_{0}^{-3} .
$$

This scaling can be understood as naturally following from the structure of eqs. (4.4), (4.5) and (4.15), although it is conceivable that it could be changed with some additional finetuning of the parameters of the theory. This relation calls nevertheless for superpotentials with derivatives satisfying $T^{n} W^{(n)} / W \sim 1$. 
Let us now try to match the coefficients (5.3) of the local expansion with an explicit superpotential of a form that may plausibly arise in these models. The simplest possibility is to try with an exponential effective superpotential that typically arises from gaugino condensation. This has the simple form $W=A e^{-a T}$, provided that $a T \gg 1$, corresponding to a weekly coupled four-dimensional low-energy effective theory. For this type of superpotential, however, one gets $T^{n} W^{(n)} / W \sim(a T)^{n}$, which is much larger than 1 as soon as $a T \gg 1$. It is then not possible to reproduce the scaling (5.4). This problem can however be cured by adding a constant term $W=\Lambda$, or possibly also a linear term $W=F T$, which may for instance arise from background fluxes 10 Notice finally that one needs a superpotential with at least 7 free parameters in order to be able to match all the local coefficients.

As a simple and 'symmetric' possibility to try out, one could consider a superpotential with a constant term plus a racetrack term for each field:

$$
W=\Lambda+A_{1} e^{-a_{1} T^{1}}+A_{2} e^{-a_{2} T^{2}}+B_{1} e^{-b_{1} T^{1}}+B_{2} e^{-b_{2} T^{2}} .
$$

This has 9 coefficients which have to satisfy 7 equations. This allows to express 7 of them in terms of the other 2 , say $b_{1}$ and $b_{2}$, and of the coefficients of the local superpotential. Among other relations, one finds that

$$
a_{i}=-\frac{b_{i} W_{i i}+W_{i i i}}{b_{i} W_{i}+W_{i i}}
$$

One can then choose the values of $b_{i}$ in such a way that $b_{i} T_{0}^{i} \gg 1$, but by eq. (5.4) one will then get $a_{i} T_{0}^{i} \sim 1$. This means that the constant term allows to make only some of the exponents in the exponential terms large, and some of them remain of order one, so that higher-power corrections may become relevant. An example of this type is obtained with the following values of the parameters, in units of $m_{3 / 2} \mathcal{V}_{\mathcal{O}}$ for $\Lambda, A_{i}, B_{i}$ and $\mathcal{V}_{\mathcal{O}}^{-2 / 3}$ for $a_{i}, b_{i}$ :

\begin{tabular}{|l|r|}
\hline$\Lambda$ & $2.63036 \times 10^{1}$ \\
$A_{1}$ & $7.37726 \times 10^{1}$ \\
$B_{1}$ & $-9.77287 \times 10^{1}$ \\
$A_{2}$ & $-1.50213 \times 10^{0}$ \\
$B_{2}$ & $-2.80545 \times 10^{0}$ \\
\hline
\end{tabular}

A more satisfactory but slightly more complicated model may be obtained by adding linear terms. Let us consider for example the following form of the superpotential:

$$
W=\Lambda+F_{1} T^{1}+F_{2} T^{2}+A_{1} e^{-a_{1} T^{1}}+A_{2} e^{-a_{2} T^{2}}+B_{1} e^{-b_{1} T^{1}}+B_{2} e^{-b_{2} T^{2}} .
$$

\footnotetext{
${ }^{10}$ This kind of effect has also been used to construct supersymmetric vacua. See for instance refs. [37,38].
} 
While one still has $W_{i i i} / W_{i i}=-a_{i}$, as this condition is unaffected by the addition of a linear term, the relation between the coefficients $a_{i}, b_{i}$ and $W_{i i i} / W_{i i}$ gets now more complicated and less constraining. This allows to find parameters such that all the exponents in the exponential terms are large. A working example of this type is obtained with the following choice of parameters, in units of $m_{3 / 2} \mathcal{V}_{\mathcal{O}}$ for $\Lambda, A_{i}, B_{i}, m_{3 / 2} \mathcal{V}_{\mathcal{O}}^{1 / 3}$ for $F_{i}$ and $\mathcal{V}_{\mathcal{O}}^{-2 / 3}$ for $a_{i}, b_{i}$ :

\begin{tabular}{|l|c|c|c|}
\hline$\Lambda$ & $-4.83093 \times 10^{-1}$ \\
$A_{1}$ & $5.14986 \times 10^{9}$ \\
$B_{1}$ & $-1.55366 \times 10^{10}$ \\
$A_{2}$ & $-4.16798 \times 10^{8}$ \\
$B_{2}$ & $2.38480 \times 10^{10}$
\end{tabular} \mid \begin{tabular}{lll|l|}
$a_{1}$ & $6.69463 \times 10^{1}$ \\
$b_{1}$ & $6.99410 \times 10^{1}$ \\
$a_{2}$ & $3.55839 \times 10^{1}$ \\
$b_{2}$ & $4.19646 \times 10^{1}$ \\
$F_{1}$ & $2.05036 \times 10^{0}$ \\
$F_{2}$ & $8.92014 \times 10^{-1}$ \\
\hline
\end{tabular}

\section{$5.2 \quad$ Heterotic models}

Let us now consider heterotic models. In this case, the way in which the dilaton and the complex structure moduli may be stabilised is less understood, but we will nevertheless assume that these do not play any role and focus on two volume moduli. As an explicit example satisfying the necessary condition $\Delta<0$, let us consider a CY manifold with intersection numbers $d_{111}=1, d_{112}=0, d_{122}=1$ and $d_{222}=0$, for which $\Delta=-108<0$. The corresponding Kähler potential is:

$$
K=-\log \left[\frac{1}{6}\left(T^{1}+\bar{T}^{1}\right)^{3}+\frac{1}{2}\left(T^{1}+\bar{T}^{1}\right)\left(T^{2}+\bar{T}^{2}\right)^{2}\right] .
$$

We chose in this case the values of the field vevs in such a way that $a_{\mathcal{H}}=9$, corresponding to setting $\hat{s}^{i}=0$. This choice does not correspond to the largest possible sGoldstino mass in this case, but it has the virtue of maintaining some similarity with the orientifold examples. Moreover, we require as before some definite numerical value $\mathcal{V}_{\mathcal{H}}$ for the volume. This leads then to the following values of the vevs, in units of $\mathcal{V}_{\mathcal{H}}^{1 / 3}$ :

$$
\begin{array}{|l|l|}
\hline T_{0}^{1} & 0.405666 \\
T_{0}^{2} & 0.749277 \\
\hline
\end{array}
$$

Applying the procedure outlined in the previous section, one finds the following set of local parameters, in units of $m_{3 / 2} \mathcal{V}_{\mathcal{H}}^{1 / 2}$ for $W_{0}, m_{3 / 2} \mathcal{V}_{\mathcal{H}}^{1 / 6}$ for $W_{i}, m_{3 / 2} \mathcal{V}_{\mathcal{H}}^{-1 / 6}$ for $W_{i i}$ and $m_{3 / 2} \mathcal{V}_{\mathcal{H}}^{-1 / 2}$ for $W_{i i i}$ : 


\begin{tabular}{|l|r|}
\hline$W_{0}$ & 1.00000 \\
$W_{1}$ & 1.64415 \\
$W_{2}$ & 2.60392 \\
$W_{11}$ & -17.4400 \\
$W_{22}$ & 3.82418 \\
$W_{111}$ & 616.732 \\
$W_{222}$ & 2.31275 \\
\hline
\end{tabular}

In this model, the four physical square-mass eigenvalues $m_{i}^{2}$ at the minimum are given by $4.43,5.94,191.89$ and 311.92 in units of $m_{3 / 2}^{2}$.

We may now proceed as for orientifold models and fit these coefficients with a superpotential involving exponential, constant or linear terms. In this case, however, the possible origin of such terms is less clear than for orientifolds. For instance, gaugino condensation produces exponential contributions, but with an exponent involving in first approximation only the dilaton. It is however common that the effective gauge coupling receives perturbative threshold corrections depending on the volume moduli as well. Assuming then that the dilaton does not play any role and the volume moduli are large, one can be left with an exponent linear in $T$. Notice moreover that taking this perspective there is no reason to require any longer that the exponent should be large and positive (see for example $[39,40])$. As a toy example, we can then again consider a superpotential of the form (5.5). Fitting the coefficients one finds the following values, in units of $m_{3 / 2} \mathcal{V}_{\mathcal{H}}^{1 / 2}$ for $\Lambda, A_{i}, B_{i}$ and $\mathcal{V}_{\mathcal{H}}^{-1 / 3}$ for $a_{i}, b_{i}$ :

$$
\begin{array}{|l|r|}
\hline \Lambda & -5.97604 \times 10^{-1} \\
A_{1} & -3.62358 \times 10^{5} \\
B_{1} & -1.46692 \times 10^{0} \\
A_{2} & 7.98841 \times 10^{-1} \\
B_{2} & 7.49672 \times 10^{-1} \\
b_{1} & 4.36876 \times 10^{1} \\
a_{2} & -1.28225 \times 10^{0} \\
b_{2} & 5.33848 \times 10^{0} \\
\hline
\end{array}
$$

\section{Conclusions}

In this paper we have developed a systematic method for constructing metastable dS vacua in supergravity models describing the volume moduli sector of CY string compactifications, without invoking subleading corrections breaking the no-scale property or uplifting terms. To do so, we have exploited the fact that there exists a necessary condition for the existence of metastable vacua, which constrains the allowed scalar geometry 
and supersymmetry-breaking directions [25]. We have focused on the simplest non-trivial case of two volume moduli, which allows for a detailed analysis, but we believe that the more complicated cases with more than two volume moduli can be treated similarly. We have singled out the special Goldstino direction which allows to maximise the moduli masses, and in the case of orientifold compactifications, we have found a strong upper bound of the lightest modulus mass as a function of the gravitino mass.

The main result of the paper is an explicit procedure allowing to construct the local form of the superpotential that gives a metastable dS vacuum in models where the Kähler potential satisfies the necessary condition for metastability on the sign of the discriminant $\Delta$ of the intersection numbers $d_{i j k}$. We have also applied this procedure to construct a few simple examples of concrete models admitting viable metastable vacua that may plausibly emerge within heterotic and orientifold string compactifications with background fluxes and gaugino condensation effects. The fact that these models need to have more than one dynamical field and at least seven independent parameters in the superpotential to allow for the construction is probably the reason why such models have not been noted earlier. It is still an open question to study more realistic, more generic or even more minimal models, but we have now a proof of existence for $\mathrm{dS}$ vacua arising from simple F-term supersymmetry breaking in both the orientifold and heterotic case.

We believe that our results emphasise in a clear way that it is actually possible to achieve genuine metastable dS vacua even in models satisfying the no-scale property, provided that the scalar geometry is sufficiently generic. This is the case for the volume moduli sector of smooth CY compactifications, as opposed to their orbifolds limits, when at least two moduli arise. But of course in order to construct a realistic model, there are several other issues to be addressed. One of them is the detailed mechanism stabilizing the other moduli and the impact of their dynamics onto the dS vacuum admitted by the volume moduli sector. Another is the life-time of the dS vacuum against decay to other supersymmetric AdS vacua that generically arise at different values of the fields [41-43].

\section{Acknowledgements}

This work was partly supported by the German Science Foundation (DFG) under the Collaborative Research Centre (SFB) 676, the Swiss National Science Foundation, and by the Netherlands Organisation for Scientic Research (NWO) under a VIDI and a VICI Innovative Research Incentive Grant. M. G.-R. and C. G. are grateful to the Institute for Theoretical Physics of EPFL for hospitality during the completion of this work. G. A. P. would like to thank A. Achúcarro for useful discussions. 


\section{References}

[1] S. B. Giddings, S. Kachru and J. Polchinski, Hierarchies from fluxes in string compactifications, Phys. Rev. D 66 (2002) 106006 [arXiv:hep-th/0105097].

[2] A. Saltman and E. Silverstein, The scaling of the no-scale potential and de Sitter model building JHEP 0411 (2004) 066 [arXiv:hep-th/0402135].

[3] V. Balasubramanian, P. Berglund, J. P. Conlon and F. Quevedo, Systematics of moduli stabilisation in Calabi-Yau flux compactifications, JHEP 0503 (2005) 007 arXiv:hep-th/0502058.

[4] D. Lust, S. Reffert, W. Schulgin and S. Stieberger, Moduli stabilization in type IIB orientifolds. I: Orbifold limits, Nucl. Phys. B 766 (2007) 68 [arXiv:hep-th/0506090]; D. Lust, S. Reffert, E. Scheidegger, W. Schulgin and S. Stieberger, Moduli stabilization in type IIB orientifolds. II, Nucl. Phys. B 766 (2007) 178 [arXiv:hep-th/0609013].

[5] S. Kachru, R. Kallosh, A. Linde and S. P. Trivedi, De Sitter vacua in string theory, Phys. Rev. D 68 (2003) 046005 arXiv:hep-th/0301240].

[6] E. Silverstein, Simple de Sitter Solutions, Phys. Rev. D 77 (2008) 106006 arXiv:0712.1196 [hep-th]].

[7] S. S. Haque, G. Shiu, B. Underwood and T. Van Riet, Minimal simple de Sitter solutions, arXiv:0810.5328 [hep-th].

[8] C. P. Burgess, R. Kallosh and F. Quevedo, de Sitter string vacua from supersymmetric D-terms, JHEP 0310 (2003) 056 arXiv:hep-th/0309187.

[9] K. Choi, A. Falkowski, H. P. Nilles and M. Olechowski, Soft supersymmetry breaking in KKLT flux compactification, Nucl. Phys. B 718 (2005) 113 [hep-th/0503216].

[10] G. Villadoro and F. Zwirner, de Sitter vacua via consistent D-terms, Phys. Rev. Lett. 95 (2005) 231602 [arXiv:hep-th/0508167]; D terms from D-branes, gauge invariance and moduli stabilization in flux compactifications, JHEP 0603 (2006) 087 arXiv:hep-th/0602120.

[11] A. Achucarro, B. de Carlos, J. A. Casas and L. Doplicher, de Sitter vacua from uplifting D-terms in effective supergravities from realistic strings, JHEP 0606 (2006) 014 [arXiv:hep-th/0601190].

[12] K. Choi and K. S. Jeong, Supersymmetry breaking and moduli stabilization with anomalous U(1) gauge symmetry JHEP 0608 (2006) 007 arXiv:hep-th/0605108. 
[13] A. Achucarro and K. Sousa, F-term uplifting and moduli stabilization consistent with Kahler invariance, arXiv:0712.3460 [hep-th].

[14] M. Becker, G. Curio and A. Krause, De Sitter vacua from heterotic M-theory, Nucl. Phys. B 693 (2004) 223 arXiv:hep-th/0403027.

[15] F. Saueressig, U. Theis and S. Vandoren, On de Sitter vacua in type IIA orientifold compactifications, Phys. Lett. B 633 (2006) 125 [arXiv:hep-th/0506181].

[16] K. Becker, M. Becker, M. Haack and J. Louis, Supersymmetry breaking and alpha'-corrections to flux induced potentials, JHEP 0206, 060 (2002) arXiv:hep-th/0204254.

[17] V. Balasubramanian and P. Berglund, Stringy corrections to Kaehler potentials, SUSY breaking, and the cosmological constant problem, JHEP 0411 (2004) 085 arXiv:hep-th/0408054].

[18] S. L. Parameswaran and A. Westphal, de Sitter string vacua from perturbative Kaehler corrections and consistent D-terms, JHEP 0610 (2006) 079 arXiv:hep-th/0602253].

[19] E. Palti, G. Tasinato and J. Ward, Weakly-coupled IIA Flux Compactifications, JHEP 0806 (2008) 084 arXiv:0804.1248 [hep-th]].

[20] M. Berg, M. Haack and E. Pajer, Jumping through loops: on soft terms from large volume compactifications, JHEP 0709 (2007) 031 arXiv:0704.0737 [hep-th]].

[21] J. A. Casas, The generalized dilaton supersymmetry breaking scenario, Phys. Lett. B 384 (1996) 103 [hep-th/9605180].

[22] R. Brustein and S. P. de Alwis, Moduli potentials in string compactifications with fluxes: Mapping the discretuum, Phys. Rev. D 69 (2004) 126006 [hep-th/0402088.

[23] M. Gomez-Reino and C. A. Scrucca, Locally stable non-supersymmetric Minkowski vacua in supergravity, JHEP 0605 (2006) 015 arXiv:hep-th/0602246].

[24] M. Gomez-Reino and C. A. Scrucca, Constraints for the existence of flat and stable non-supersymmetric vacua in supergravity, JHEP 0609 (2006) 008 arXiv:hep-th/0606273.

[25] L. Covi, M. Gomez-Reino, C. Gross, J. Louis, G. A. Palma and C. A. Scrucca, de Sitter vacua in no-scale supergravities and Calabi-Yau string models, JHEP 0806 (2008) 057 arXiv:0804.1073 [hep-th]]. 
[26] L. Covi, M. Gomez-Reino, C. Gross, J. Louis, G. A. Palma and C. A. Scrucca, Constraints on modular inflation in supergravity and string theory, JHEP 0808 (2008) 055 [arXiv:0805.3290 [hep-th]].

[27] M. Gomez-Reino, J. Louis and C. A. Scrucca, No metastable de Sitter vacua in N=2 supergravity with only hypermultiplets, arXiv:0812.0884 [hep-th].

[28] M. Gomez-Reino and C. A. Scrucca, Metastable supergravity vacua with $F$ and $D$ supersymmetry breaking, JHEP 0708 (2007) 091 [arXiv:0706.2785 [hep-th]].

[29] K. Choi, A. Falkowski, H. P. Nilles, M. Olechowski and S. Pokorski, Stability of flux compactifications and the pattern of supersymmetry breaking, JHEP 0411 (2004) 076 arXiv:hep-th/0411066|.

[30] S. P. de Alwis, Effective potentials for light moduli, Phys. Lett. B 626, 223 (2005) arXiv:hep-th/0506266.

[31] A. Achucarro, S. Hardeman and K. Sousa, F-term uplifting and the supersymmetric integration of heavy moduli, JHEP 0811 (2008) 003 [arXiv:0809.1441 [hep-th]]; Consistent Decoupling of Heavy Scalars and Moduli in N=1 Supergravity, Phys. Rev. D 78 (2008) 101901 [arXiv:0806.4364 [hep-th]].

[32] D. Gallego and M. Serone, An Effective Description of the Landscape - I, arXiv:0812.0369 [hep-th].

[33] T. W. Grimm and J. Louis, The effective action of $N=1$ Calabi-Yau orientifolds, Nucl. Phys. B 699, 387 (2004) arXiv:hep-th/0403067.

[34] R. D'Auria, S. Ferrara and M. Trigiante, c-map, very special quaternionic geometry and dual Kaehler spaces, Phys. Lett. B 587 (2004) 138 arXiv:hep-th/0401161; Homogeneous special manifolds, orientifolds and solvable coordinates, Nucl. Phys. B 693 (2004) 261 arXiv:hep-th/0403204].

[35] G. D. Coughlan, W. Fischler, E. W. Kolb, S. Raby and G. G. Ross, Cosmological problems for the Polonyi potential, Phys. Lett. B 131 (1983) 59.

[36] J. Gray, Y. H. He and A. Lukas, Algorithmic algebraic geometry and flux vacua, JHEP 0609 (2006) 031 [hep-th/0606122]; J. Gray, Y. H. He, A. Ilderton and A. Lukas, A new method for finding vacua in string phenomenology, hep-th/0703249.

[37] A. Micu, E. Palti and G. Tasinato, Towards Minkowski vacua in type II string compactifications, JHEP 0703, 104 (2007) arXiv:hep-th/0701173]. 
[38] E. Palti, Low energy supersymmetry from non-geometry, JHEP 0710, 011 (2007) arXiv:0707.1595 [hep-th]].

[39] M. Serone and A. Westphal, Moduli stabilization in meta-stable heterotic supergravity vacua JHEP 0708 (2007) 080 arXiv:0707.0497 [hep-th]].

[40] M. Badziak and M. Olechowski, Volume modulus inflection point inflation and the gravitino mass problem, arXiv:0810.4251 [hep-th].

[41] S. R. Coleman and F. De Luccia, Gravitational effects on and of vacuum decay, Phys. Rev. D 21 (1980) 3305.

[42] S. Weinberg, Does gravitation resolve the ambiguity among supersymmetry vacua?, Phys. Rev. Lett. 48 (1982) 1776.

[43] T. Banks, Heretics of the false vacuum: Gravitational effects on and of vacuum decay. II, arXiv:hep-th/0211160. 